\title{
Neue Wege für Holztragwerke Das Forschungslabor IBOIS an der EPF Lausanne
}

Yves Weinand

Das IBOIS Die in den letzten zwei Jahrhunderten bestehende Vorherrschaft von zunächst Stahl, später dann Stahlbeton, in der Forschung und der Praxis der Bauingenieurwissenschaft und Werkstoffkunde hat dazu geführt, dass eine große Forschungslücke in Bezug auf Holz als Konstruktionsmaterial entstanden ist. Das intuitive Wissen von Zimmerleuten und unseren beruflichen Vorgängern ist verloren gegangen, seit sich im 18. Jahrhundert der Beruf des Ingénieur des Ponts et Chaussées (Bauingenieur) entwickelt hat, der Holz nicht als Baumaterial nutzt, weil er ihm a priori einen geringeren Stellenwert zuweist als Stahl und Beton.

Mein duales Profil als Architekt und Bauingenieur ermöglicht es mir, den Fokus auf die interdisziplinären Aspekte des Bauentwurfs zu richten, und so Synergien zu entwickeln. Da ich wegweisende Forschungsarbeit sowohl in der Baustatik als auch in der Konstruktion durchgeführt habe, unterscheidet sich meine Sichtweise einiger Phänomene deutlich von der Perspektive der meisten Theoretiker oder Praktiker, die nur auf eines dieser beiden Gebiete spezialisiert sind. Durch meine singuläre Position als jemand, der aktiv in der Praxis und in der Forschung tätig ist und beides auch lehrt, hat meine breite Erfahrung mir ein Gleichgewicht ermöglicht, in welchem von den Architekten beanspruchte Werte wie Subjektivität und Ästhetik einem umfassenden baustatischen und technischem Wissen gleichwertig gegenüber stehen, wodurch diese Werte eher verstärk als abgeschwächt werden. Meine Forschungsarbeit konzentriert sich auf technische, konstruktive, werkstoffbezogene und baustatische Aspekte - die, mit einigen Ausnahmen seit Leonardo da Vincis Zeit, von Architekten auf der Suche nach Verwirklichung ihrer ästhetischen Ansprüche allzu sehr vernachlässigt oder delegiert worden sind. Sie berücksichtigt unzählige grundlegende Verknüpfungen zwischen Kunst und Wissenschaft ebenso wie die spezifischen Zwänge der beobachteten Phänomene und 
deren konkreter Umsetzung. Auswirkungen des Maßstabs werden im Bereich der strukturellen Analyse für die Baukonstruktion häufig einfach ignoriert. Mein Ansatz nimmt die mechanischen Anforderungen von Form/Struktur als Attribute wahr, die nur im Rahmen des geometrisch skalierten Phänomens, von dem sie abhängig sind, volle Bedeutung und Sinn erlangen können. Ich betrachte die Entstehung der digitalen Darstellung von Architektur als unschätzbares Werkzeug, das jedoch nur eingesetzt werden kann, um die Integration von Struktur, Form und Materia innerhalb unseres Entwurfsbegriffs zu stärken, wenn die physische Realität jedes beobachteten Phänomens als Gesichtspunkt von größter Bedeutung behandelt wird, sodass die Herstellung von Form und Raum mit derjenigen von Struktur miteinander verknüpft wird.

Forschung in Architektur und Bauingenieurwissenschaft Die architektonischen Forschungs-, Kompositions-, Produktions- und sogar Konstruktionsprozesse bleiben eng verknüpft mit den persönlichen Entwurfsprozessen des einzelnen Architekten, und die Ausdrucksfreiheit des Architekten als Künstler wird per definitionem als inhärenter Bestandteil des kreativen Prozesses respektiert. Diese epistemologische Rahmen macht die architektonische Forschung andersartig und schwer akzeptierbar für Disziplinen, die primär in der Kultur der Technik oder de Gesellschaftswissenschaften verwurzelt sind. Im Allgemeinen zielt die Forschung in der Architektur nicht primär darauf ab, der angewandten Technik Geltung zu verschaffen. Wahrhaft interdisziplinäre Forschungsansätze, die die Architektur mit der Bauingenieurwissenschaft verknüpfen, sind nach wie vor wenig verbreitet. Technische Gesichtspunkte werden sehr häufig als quasi neutrales Wissen betrachtet, das den ursprünglichen kreativen Entwurfsprozess eines einzelnen Architekten nicht in einer festgelegten Weise beeinflusst oder beeinflussen sollte. Technische Verfahren, Bauausführungsverfahren und schließlich Gesichtspunkte der Baustatik und der Bautechnik gelten in manchen Fällen fast als unwillkommene Faktoren. Häufig werden diese angeblich neutralen technischen Aspekte erst in einem späteren Stadium des Entwurfsprozesses berücksichtigt, wodurch die wirklich interdisziplinäre und fundamentale Qualität aufs Spiel gesetzt wird, die solche Forschungsansätze verfolgen könnten. Selbst einige gefeierte, ikonenhafte Bauwerke, wie z. B. das Guggenheim-Museum in Bilbao von Frank O. Gehry oder das Olympiastadion in Peking von Herzog \& de Meuron, zeigen, wie der Formalismus das bautechnische Konzept zu einer zweitrangigen Frage degradiert.

Tragwerke und die elementaren Bestandteile größerer integraler Einheiten wie Balken, Stützpfeiler und Bauelemente müssen in erster Linie robust sein, um ihre tragende Qualität zu erlangen. Unsere heutige Gesellschaft assoziiert große Ingenieurbauten nicht mit Begriffen wie "Textil" oder "Holz". Für die meisten Menschen verbindet sich mit dem Begriff "Textil" die Vorstellung der Weichheit, was mit dem allgemeinen Kontext von Bauwerken unvereinbar erscheint. Der Begriff "Textil" umfasst zwar eine Vielzahl verschiedener Anwendungen und Interpretationen, es hat jedoch bislang keine Versuche gegeben, seine Eigenschaften und
Herstellungstechniken auf den Bereich der Holzkonstruktion zu übertragen. Doch mit der Strategie, textilähnliche (gewobene oder geflochtene) Holzstrukturen zu verwenden, lässt sich die faserige, von Natur aus flexible Beschaffenheit des Holzes, die in den vergangenen zwei Jahrhunderten als Einschränkung wahrgenommen wurde, nutzen und in einen konstruktiven Vorteil umkehren. Die Entwicklung von Holzkonstruktionen, welche auf textile Strukturen aufbauen, artikuliert sowoh eine Vision der Zukunft als auch ein Verständnis der Vergangenheit. Sie ist von der Vision des Bauens als einem integrierten Entwurfsprozess inspiriert, in dem Aspekte des Handwerks, der Technik, der Ästhetik und der Baustatik zusammenkommen wie dies vor der Zeit der Aufklärung der Fall war - nur dass dieses Mal technische Verfahren und Werkzeuge der heutigen Zeit zur Anwendung kommen.

Das zur Diskussion stehende Rohmaterial besitzt von Natur aus Eigenschaften (wie z. B. Glätte), die auch die von Architekten gesuchten ästhetischen und konzeptionellen Qualitäten bieten können. Die neu entstehenden digitalen Entwurfswerkzeuge für Architektur und die Art und Weise, in der digitale Zeichenprogramme nun als Instrumente für das Begreifen von Architektur gesehen werden, hat den Weg für breitere Anwendungen der digitalen Technik eröffnet, einschließlich technischer Anwendungen: Technische Aspekte können ins Entwurfswerkzeug eingebettet werden. Technische Fortschritte, die heute in Reichweite liegen, ermöglichen die Integration textiler Grundprinzipien, textiler Technologien und Fertigungssysteme auf eine Weise, die noch vor einigen Jahren undenkbar war.

Die Umweltargumente, die für eine stärkere Nutzung des (erneuerbaren) Rohstoffs Holz sprechen, sind unbestreitbar. Das wachsende gesellschaftliche Bewusstsein für die dringende Notwendigkeit, nachhaltige Baumaterialien zu verwenden, ist in den letzten Jahren zu einem wichtigen Faktor für die zurückkehrende wirtschaftliche Bedeutung der Holzbauweise geworden. Umweltaspekte tragen dazu bei, die legitime Nutzung von Holz in den Bauwerken unserer Städte wieder einzuführen oder zu festigen, und zwar in einem Ausmaß, das über viele Jahrhunderte nich vorhanden war. Wir entdecken erst jetzt, dass viele Techniken, vom Reibschweißen bis zum Stricken, Weben und sogar Origami, auch auf Holzkonstruktionen anwendbar sind. Die Arbeit meiner eigenen Gruppe zeigt gegenwärtig bereits, dass die Anwendung solcher Verfahren den Umfang der technischen und ästhetischen Attribute von Holz radikal erweitern kann. Diese Verfahren ermöglichen uns, Holz erzeugnisse zu entwickeln, die für neuartige Zwecke geeignet sind, weil unsere Gesellschaft kulturell und ökonomisch an einem Punkt angelangt ist, an dem sie Holz als Baumaterial uneingeschränkt zu akzeptieren bereit ist. Wir sehen signifikante Vorteile in der Anwendung solcher Techniken voraus, weil durch sie der Bau großer, frei geformter Konstruktionen aus kleinen, sich wiederholenden Einheiten erleichtert werden dürfte - und dadurch öffnet sich der Weg zu einer stärkeren Nutzung von Schnittholz wie auch von recycelten Holzerzeugnissen als hochwertigen Baumaterialien.

Die allmähliche Ablösung von Holz durch Stahl und Beton, die sich in den letzten zweihundert Jahren vollzogen hat, hat nicht dazu beigetragen, neue und zeitge- 
- Eine Forschungsrichtung des IBOIS ist die Entwicklung von geflochtenen Strukturen. den Prinzipien des Flechtens wird ein Maßstabsprun genommen, um dieselben Prinzipien im Maßstab von Gebäuden oder Tragwerke zur Anwendung zu bringen
Hier ist die Beschreibung Hier ist die Beschreibung tern notwendig. Markus

Zeichnungen solche moggli-

" Der hier dargestellte Fügungsprozess des Textilchanisch komplexe Situation entstehen. Das Modul besitzt Eigenspannungen, die hen und teilweise sich selbst hen und teilveise sich selbst Tormveränderung auf das Anwirkt somit als interaktives System indem es die Eigenpasst Der Werkstoff Holk scheint auf Grund seiner im Bereiche großer Ver-

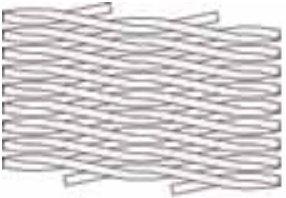

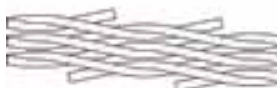

$$
5
$$

$\leadsto$ SEs

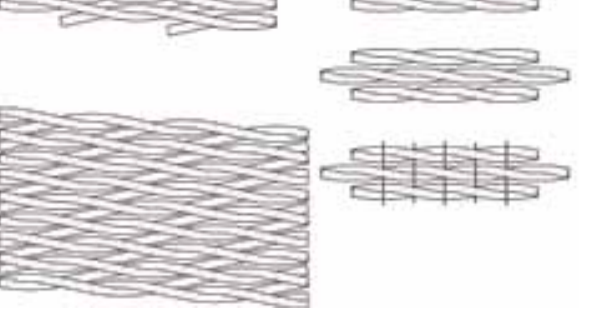

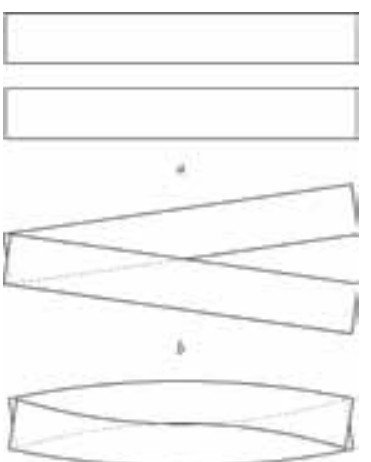

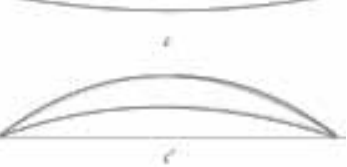

mäße Anwendungen von Holzkonstruktionen unter architektonischer und bautechnischer Perspektive zu fördern. Erst wenn man sich mit Holz intensiver befasst, als dies üblicherweise bei seiner alltäglichen Verwendung im Hochbau der Fall ist, zeigt es seine überraschend enge Verbindung mit Textilmaterialien und sein enormes Potenzial für die Anwendung textiler Techniken. Holz kann als weiches wie auch als viskoses Material mit glatten Eigenschaften klassifiziert werden. Es hat einen "Fluss", fast wie ein flüssiges Material. Holz besteht grundsätzlich aus unzähligen Zellulosefasern. Diese glatten Fasern sind biegsam und ermöglichen Kurvenformen. Diese Eigenschaften bewirken, dass großmaßstäblich verwobene flexible Holztragwerke in Bezug auf ihre Stabilität gegenüber seismischen Erschütterungen, extremen Windverhältnissen oder Schneelasten außergewöhnlich leistungsfähig sein dürften. Das Potenzial, das Holzkonstruktionen mit Webstruktur im Hochbau besitzen, um das Einsturzrisiko von Tragwerken bei solchen Herausforderungen erheblich zu senken, ist bisher noch nicht systematisch untersucht worden. Auf einer breiteren Ebene werden die vom Labor IBOIS durchgeführten Untersuchungen dazu beitragen, ein tieferes Verständnis räumlicher Strukturen im Allgemeinen entstehen zu lassen und neue Präzedenzfälle für die kooperative Interaktion zwischen Architekten und Bauingenieuren bei der Analyse dieser Strukturen zu schaffen.

Fallstudie 1: Textilmodul-Anwendungen Die hier gezeigten empirischen Modelle sind von Markus Hudert am IBOIS entwickelt worden. Anfangs verwende er Zeichnungen, um Flechtmuster zu generieren. In diesem Fall haben die ersten Zeichnungen ein sehr interessantes Konstruktionsmodul hervorgebracht. Auch wenn dieser erste Ansatz geometrische Formen steuert, enthüllt er doch neben seinen formalen Qualitäten erstaunliche strukturelle Aspekte.

Diese Konstruktion gewinnt an statischer Höhe, wenn sie belastet wird. Sie ist also eine selbst reagierende Konstruktion, und die zentrale Frage lautet daher: Nachdem wir beobachtet haben, dass diese spezifische Konstruktion an statischer Höhe
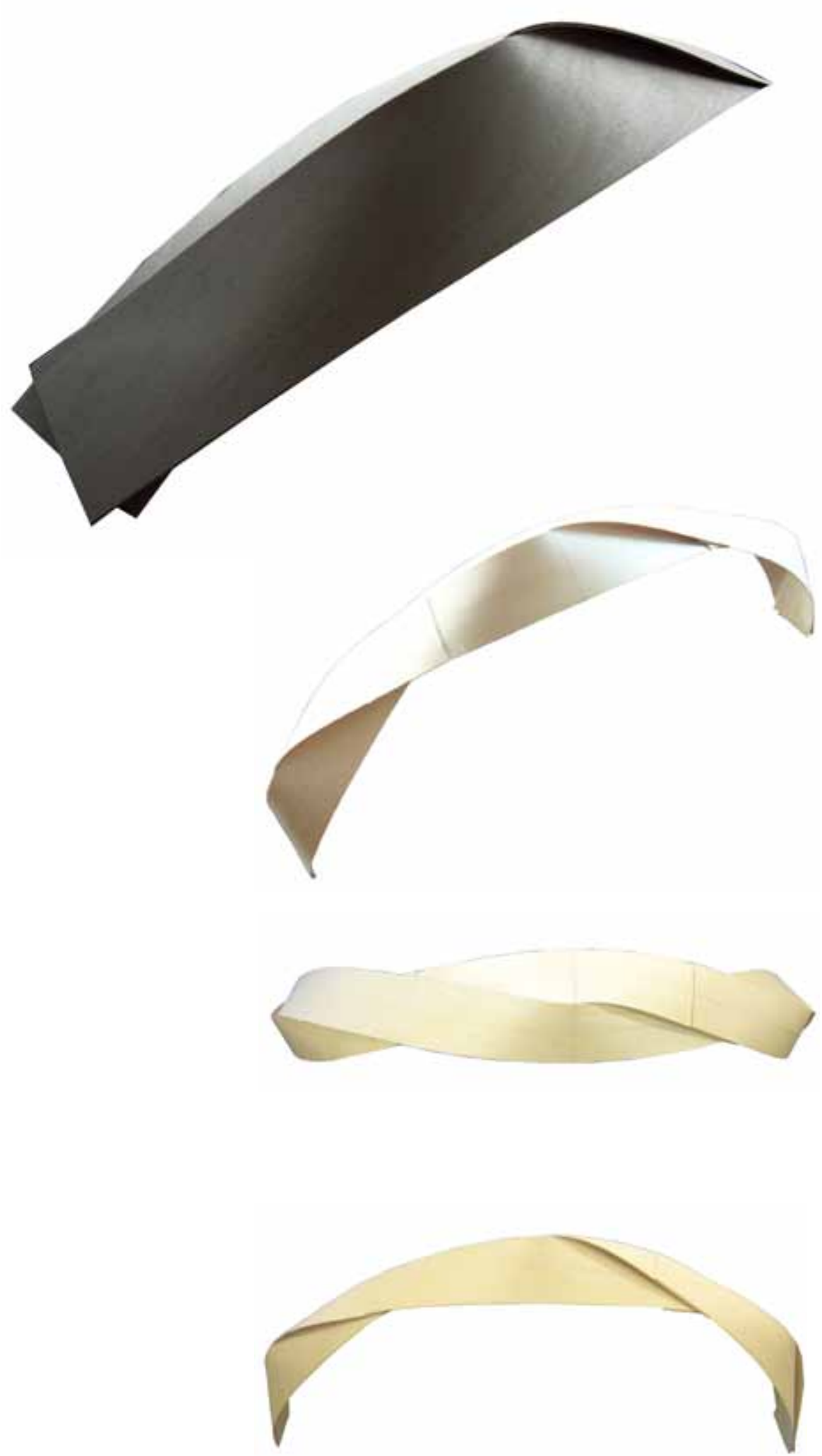
D Die beiden Hauptwebrichtungen werden aus Holz platten unterschiedicher Làngen angefertigt. Die Kon-
tinuität dieser Platten kann men gewährleistet werden gilt der geometrischen Stuation an den Überlagerungs-
punkten. An diesen Kreupunkten. An diesen Kreu-
zungspunkten besteht ebenfalls die Möglichkeit, eine inte Konstru zurichten, die sowohl die ten miteinander zu verbinden hilt, und ebenfalls erreichen.

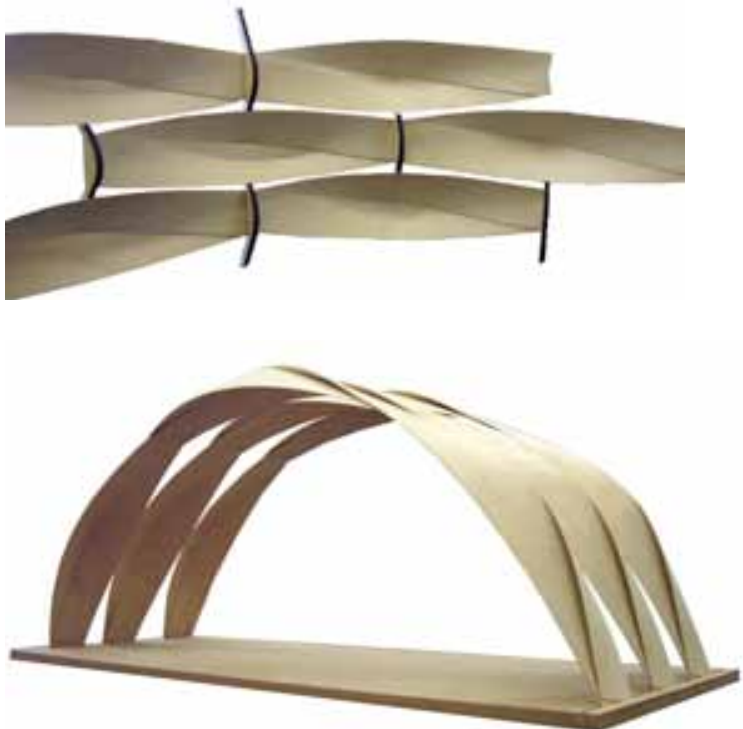

- Die Proportionen der Platten spielen eine wesentliche Rolle bei der Ausbildung des Ausrichtung wird hier in der Versuchshalle der EPFL geund Testerg verglichen

Kontaktpunkte und Randbedingungen der Konstruktion sind geometrisch und $\mathrm{me}$ einzelnen Prototypen weren unterschiedliche Auflagerund Verbindungsbedingunen entworfen und gebaut. Die Art der Ausbildung be
mi direkt den Eigenspanstimmt direkt den Eigenspanals die Art und Weise, wie das Flechtwerk "weitergeflochten" werden kann.

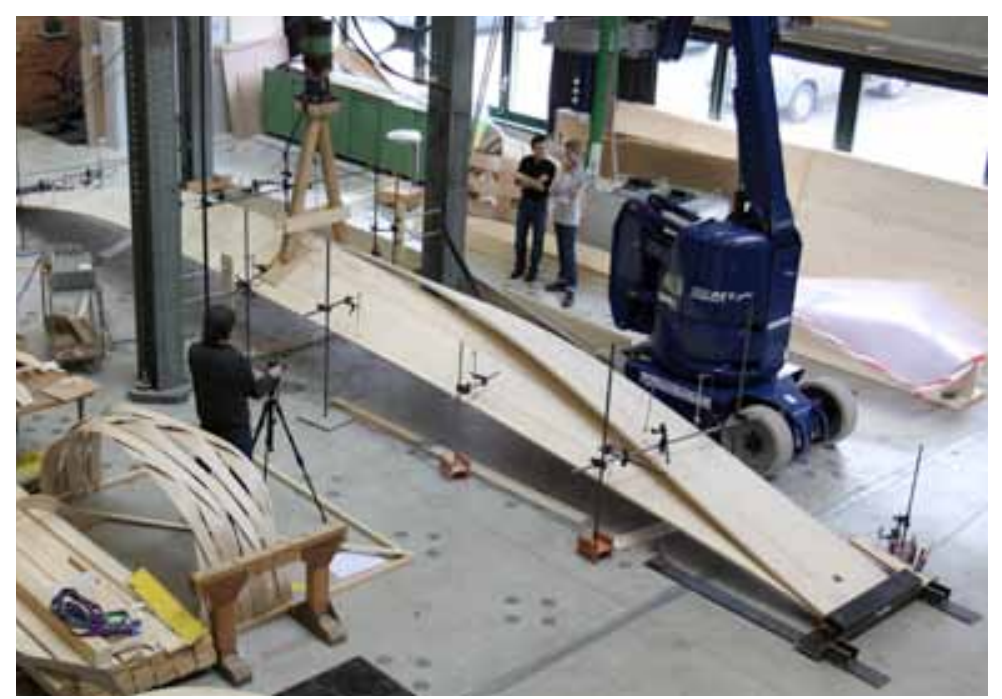

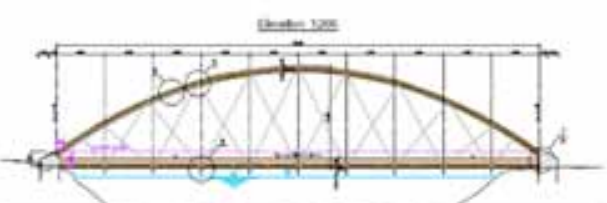
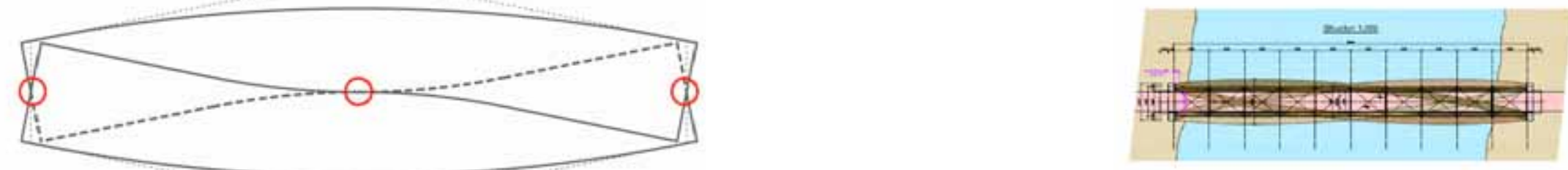

gewinnt, wenn sie sich unter experimentellen Bedingungen mit zunehmender Belastung verformt, können wir dann davon ausgehen, dass sie im Falle extremer Belastung, wie z. B. einem Sturm oder Erdbeben, ihre Disposition und ihre Festigkeit ausreichend anpasst, um solchen extremen Belastungen standzuhalten? Insbesondere ist Forschungsarbeit bezüglich der Anfangsspannungsanalyse großer Verformungen und des nicht linearen Verhaltens notwendig.

Fallstudie 2: Experimentelles Gewölbe mit Überlappung Auf der Basis dieser geometrischen Formen wurde eine aus planen Elementen bestehende Gewölbekonstruktion in digitaler Form definiert. Dieses Gewölbe, das anfangs aus einer ganzen Reihe von Elementen unterschiedlicher Größe bestand, wurde an schließend so umgearbeitet, dass es nun nur noch zwei verschiedene, sich überlappende Grundelemente verwendet.

Diese Arbeit erscheint vielversprechend, weil sie den Weg zur Beantwortung folgender Detailfragen aufzeigt: Wie sollten die überlappenden Verbindungen in großem Maßstab gebaut werden? Hinter dieser Frage verbirgt sich noch eine tiefer gehende Frage bezüglich dieser Webstruktur: Da das globale Modell direkt vom lokalen Verhalten und mechanischen Modell dieser Verbindung abhängt, wie sollte diese Konstruktion dimensioniert werden? Letzten Endes führt die Interaktion zwischen dem Globalen und dem Lokalen zu Fragen der Machbarkeit solcher großmaßstäblicher Konstruktionen und deren potenzieller Anwendung (oder auch nicht). Ebenso ist klar geworden, dass die Beziehung zwischen dem Globalen und dem Lokalen nur über die Planung der Verbindungsdetails erfolgreich gesteuert werden kann. Somit kommt der hier erörterten Zusammenarbeit zwischen Architekten und Ingenieuren ebenfalls entscheidende Bedeutung zu, weil diese bei der Definition der Verbindungsdetails interagieren müssen.
'Das Prinzip des Flechtens wurde auf eine Bogenbrücce gewendet. Vier flachliegende rechteckige Bogenqueruberschneiden sich und seitig. Die Knicklänge jedes einzelnen Bogens wird hiermit stark verringert. Durch diese besondere geo-
metrische Disposition entsteht ein Raum auf der Brücke. 
Fallstudie 3: Aussichtsturm Steve Cherpillod hat einen Turm entwickelt, der nur aus einem einzigen, höchst spezifischen Holzmodul besteht. Seine Steuerung der allgemeinen Geometrie und der globalen Form dieses Turms ermöglichte ihm, diese Komplexität zu „reduzieren“ und jenes Grundmodul zu definieren. Wieder ist sein erster Ansatz ein geometrisches Verständnis der Interaktion zwischen Treppe und Turm, welches ein sehr altes, verführerisches Thema ist. Nachdem er die räumlichen und funktionalen Anforderungen einer Treppe verstanden und sie erfolgreich in Beziehung zu den Konstruktionsanforderungen eines Turms gesetzt hatte, gelang ihm die Synthese dieser beiden Hauptaspekte des hier gezeigten Entwurfs, indem er das Grundmodul steuerte. Weitere Konstruktionsanalysen haben gezeigt, dass dieses Modul Gegenstand ausführlicher Diskussionen über seine Stabilität und die weitere Entwicklung des Turmes insgesamt sein wird.

Schlussfolgerung Diese Forschung unterliegt nicht den Zwängen der unmittelbaren praktischen Anwendung. Das Labor IBOIS verwendet Zeit und Energie darauf, unbekannte Wege zu erkunden, die nicht direkt den Anforderungen der Umsetzbarkeit oder Effizienz ausgesetzt sind, wie sie in den Ingenieurwissenschaften bestehen. Wir führen unsere Forschungsarbeit frei von den realen Zwängen oder Anforderungen durch, denen ein Bauwerk entsprechen müsste. Die hier beschriebene Forschung kann als potenziell anwendbar auf die Architektur und die Bauingenieurwissenschaft aufgefasst werden.

- Masoud Sistaninia moda lisiert die Geometrie unter Berucksichtigung der
erforderlichen hohen Veformungen und mithilfe des Finiten-Elemente-Programms Abaqus. Da sich der verformte Zustand geometrisch sehr vom unver-
formten Zustand unterscheidet, ist eine grundsätzliche edingung der Baustatik nich mehr respektiert.
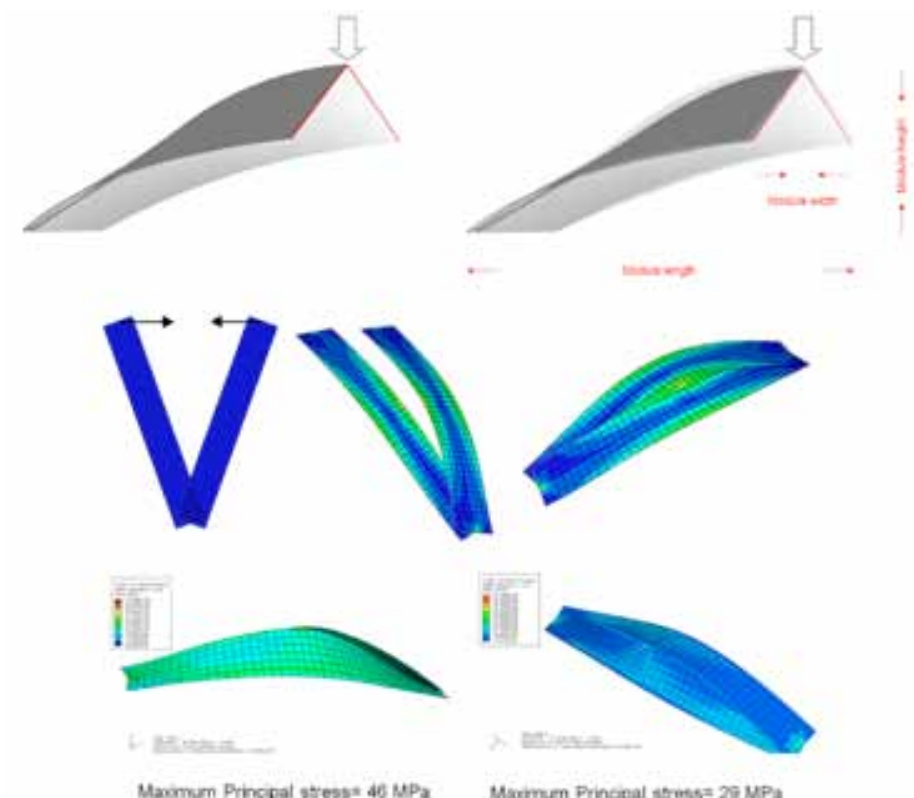

Marmen Principal atoss= 20 MPa

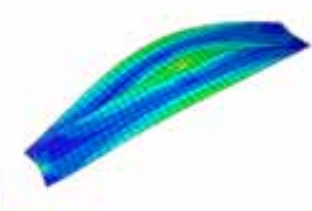

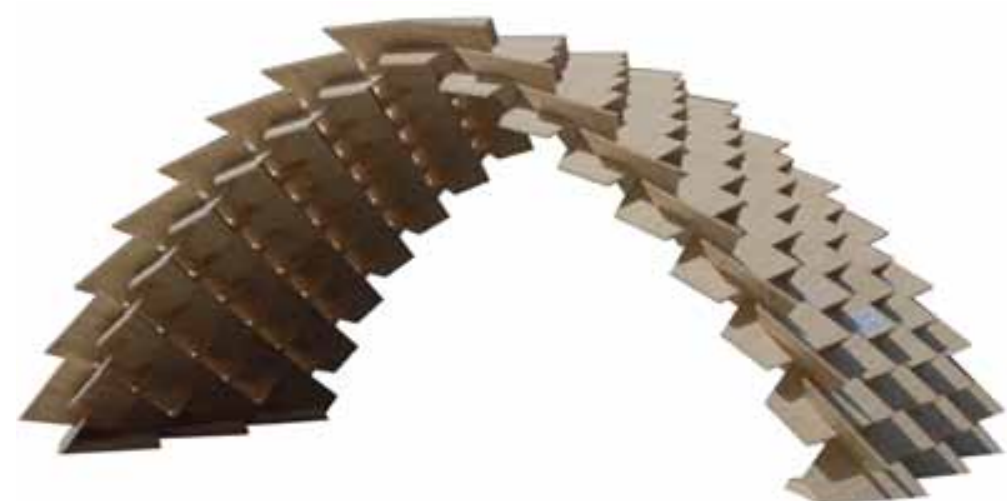

- Bei der Entwicklung der Geometrien sind beide

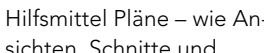
sichten, Schnitte und
Axonometrien - und auch digitale und physische Modelle von Wichtigkeit.

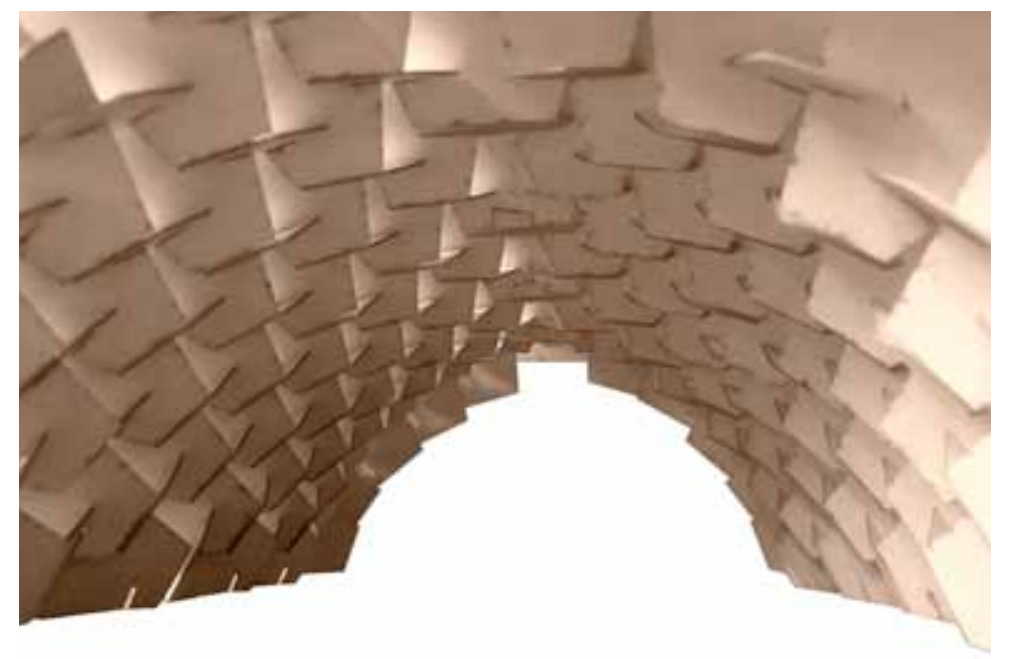

Modellansicht einer parametrisierten Bogenkonstrukvon Facetten besteht. Der Einschiebwinkel einer Facette zur nächsten kann beliebig eingestellt werden. Dieser Geometrie, aber ebenfalls die lokale Knotenausbildung. Bastien Thorel entwickelte diese Struktur im Rahmen ener Übung des Atelier Wei-

- Eine weitere Variante als Kartonmodell ausgebildet
Der Entwurfsprozess wird iterativ gesteuert. 
Neue Wege für Holztragwerke
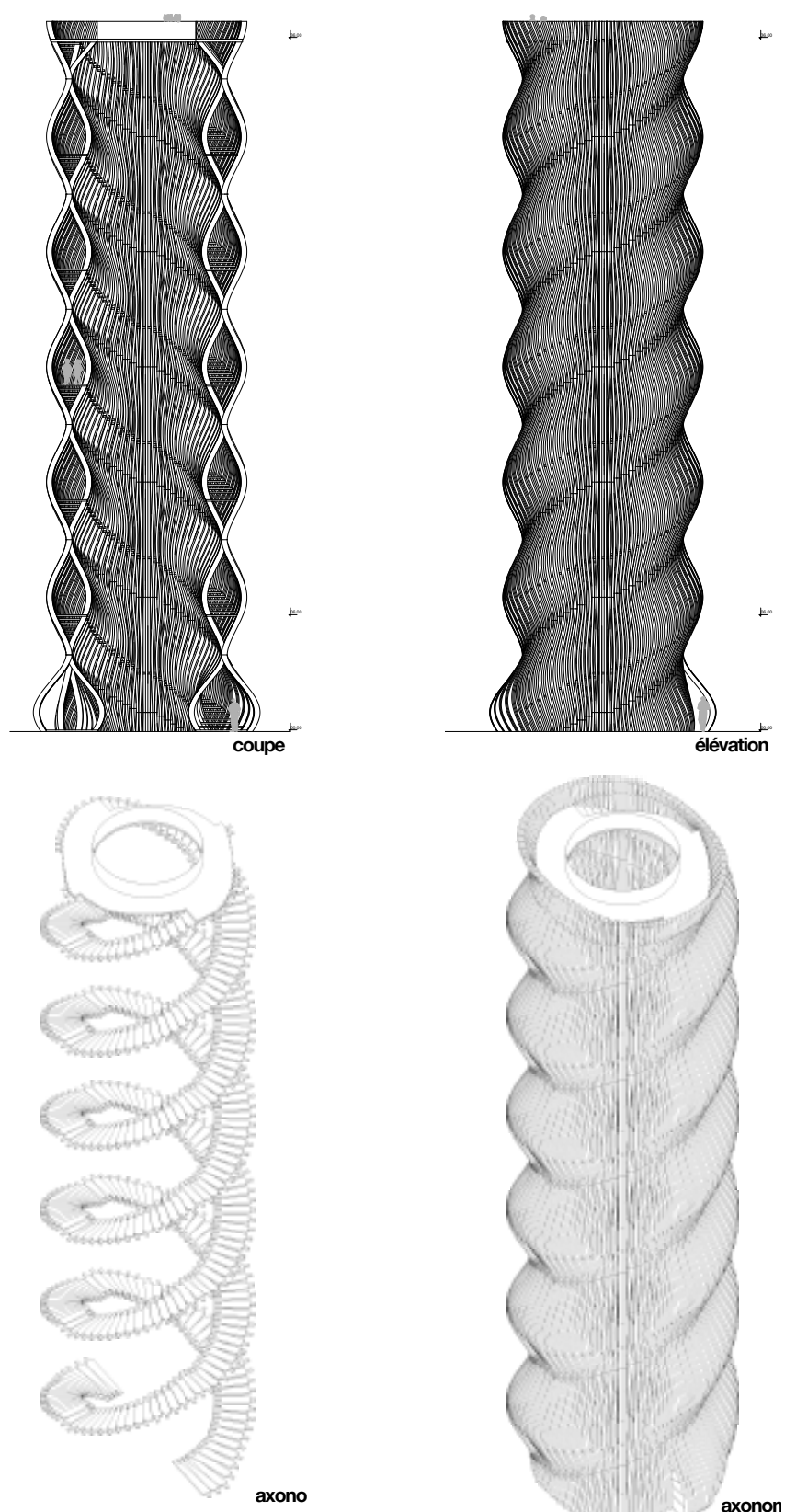

axon
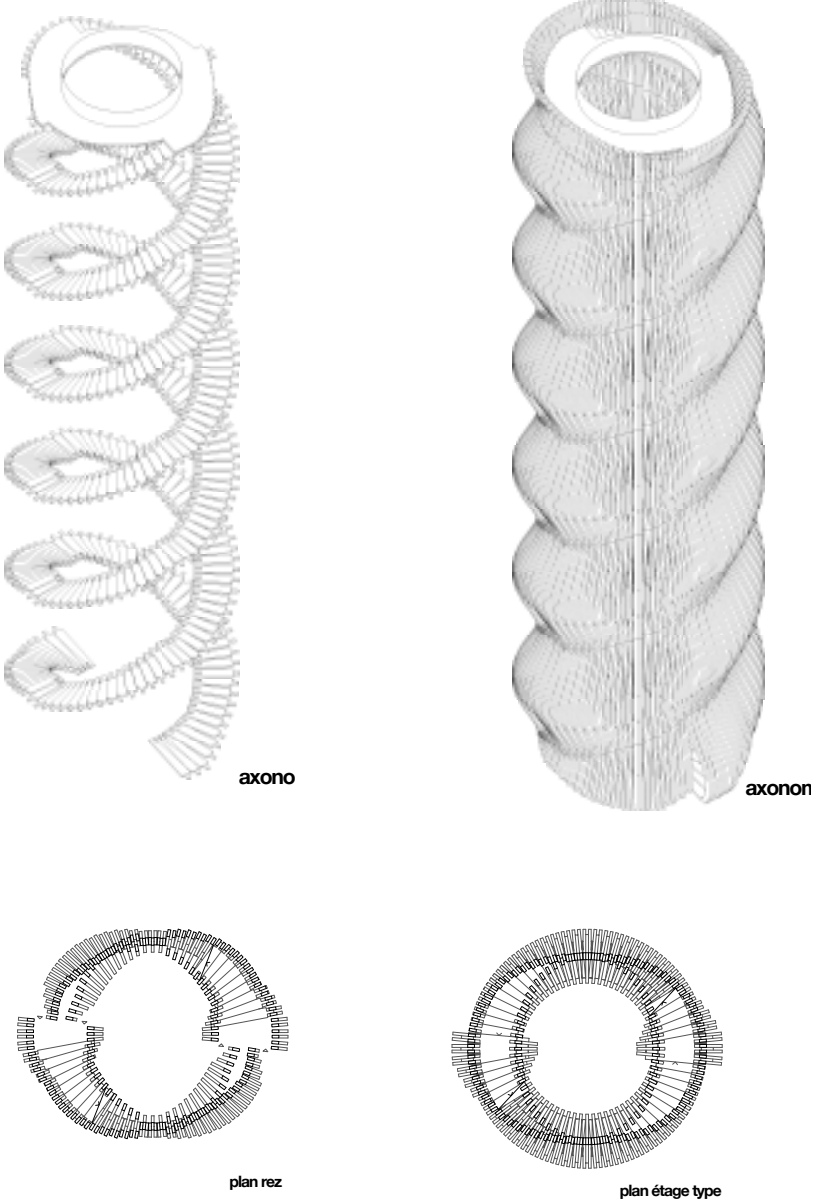

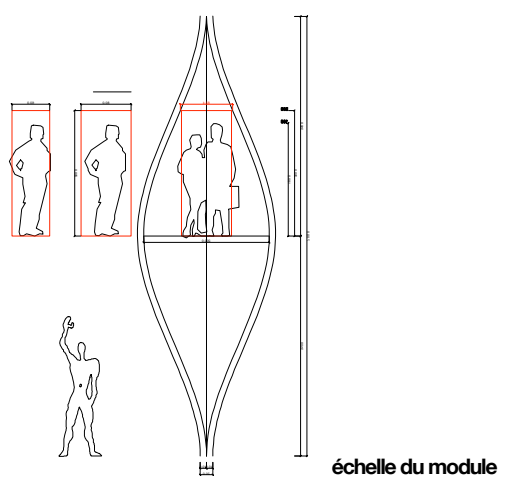

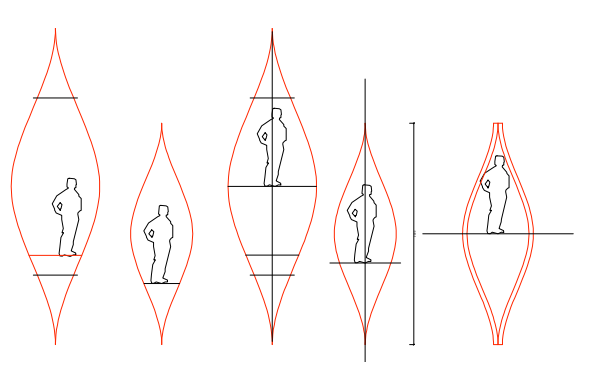

position de la marche

Alle Abbildungen auf diese Doppelseite:

Ein $35 \mathrm{~m}$ hoher Turm wurde

men Steve Cherpillodim Rah-

entworfen. Das Tragwerk des

te Treppenkonstruktion

bilden ein Ganzes. Das Bass-

Mal eingesetzt. Die ingenie

mässige Betrachtung des

es hat ergeben, dass di

Verbindung und Aussteifung

dieses Basiselement dar-

lung des Turmes muss dieses

Element optimiert werden.

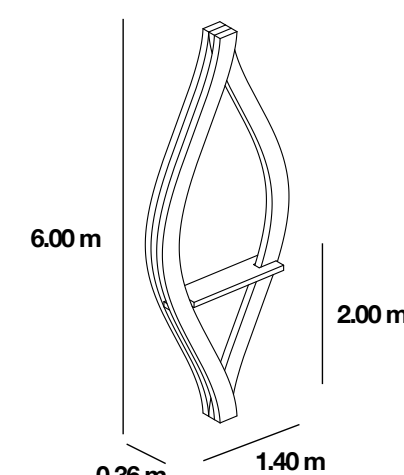

$0.36 \mathrm{~m}$

$2.00 \mathrm{~m}$

$1.40 \mathrm{~m}$

dimension du module
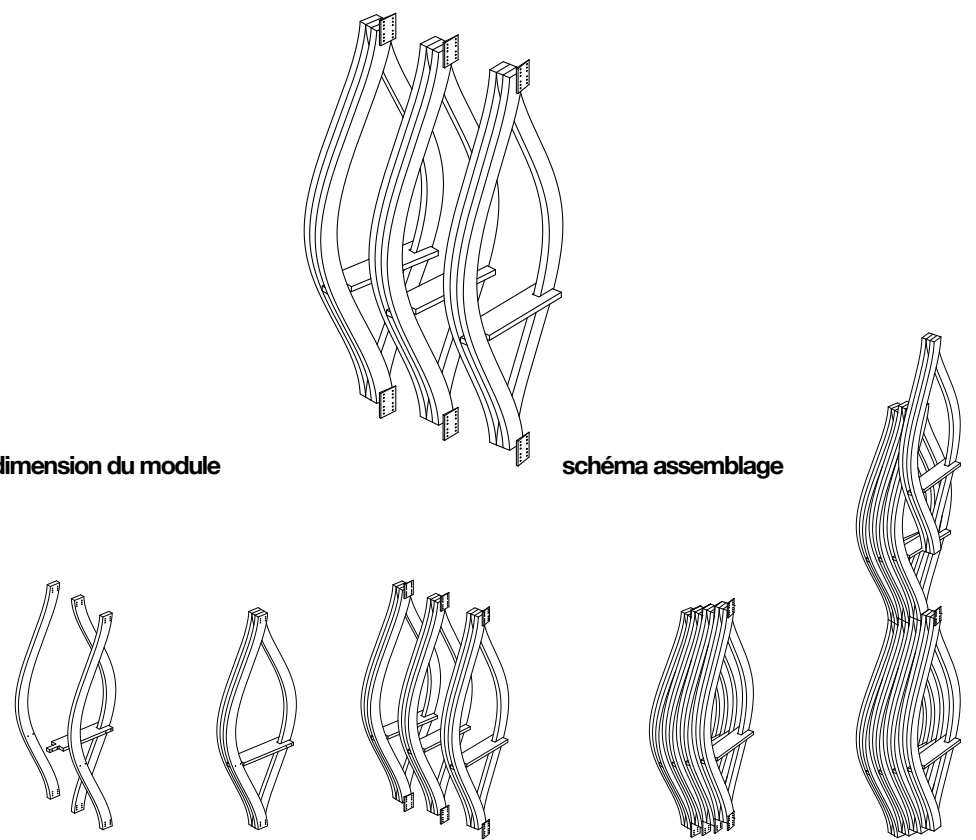